\title{
Caracterização dos pacientes portadores de insuficiência renal atendidos na emergência de um hospital geral
}

\section{Characterization of patients with renal failure treated at a general hospital emergency department \\ Caracterización de los pacientes portadores de insuficiencia renal tratados en el servicio de urgencias de un hospital general}

\author{
Eduardo Tavares Gomes'; Mônica de Melo Macêdo Papaléo II
}

\begin{abstract}
RESUMO: As unidades de saúde que prestam atendimento de emergência devem estar preparadas para o atendimento a pacientes em insuficiência renal aguda, visto que o diagnóstico, escolha do método dialítico, preparo do paciente e acesso venoso adequado precoces são decisivos. Objetivou-se caracterizar os pacientes atendidos em uma emergência geral com insuficiência renal aguda. Trata-se de um estudo descritivo, retrospectivo, de abordagem quantitativa, de dados de 78 prontuários de pacientes atendidos em julho e agosto de 2012, na emergência do Hospital Otávio de Freitas, Recife, Pernambuco. As queixas que motivam os pacientes a procurarem a emergência podem ser diversas e inespecíficas em relação à patologia renal, mas a investigação após a admissão finda por indicar o grau de lesão e indicar a necessidade de tratamento dialítico. Um grupo significativo chega aos serviços de emergência já com perda da função renal ou em estágio terminal de doença e necessidade de transplante renal. Palavras-Chave: Enfermagem; nefrologia; emergência; insuficiência renal.
\end{abstract}

\begin{abstract}
Health facilities that provide emergency care must be prepared to handle patients in acute renal failure, since early diagnosis, choice of dialysis method, patient preparation and suitable venous access are crucial. This study characterized patients with acute renal failure treated at an general hospital emergency department. This retrospective, quantitative, descriptive study examined data from the medical records of 78 patients treated in July and August 2012 at the Emergency Unit of Otávio de Freitas Hospital, Recife, Pernambuco State. The complaints that bring patients to emergency services can be diverse and unspecific as regards renal pathology, but the post-admission investigation ends by indicating the degree of injury and need for dialysis. A significant number arrive at emergency services already with loss of renal function or end-stage disease, and needing a kidney transplant.

Keywords: Nursing; nephrology; emergency; acute renal failure.
\end{abstract}

RESUMEN: Los centros de salud que brindan atención de urgencia deben estar preparados para atender a los pacientes con insuficiencia renal aguda, ya que el diagnóstico, la elección del método dialítico, la preparación del paciente y el acceso venoso adecuado tempranos son cruciales. Este estudio tuvo como objetivo caracterizar a los pacientes con insuficiencia renal aguda atendidos en el servicio de urgencias de un hospital general. Se trata de un estudio descriptivo, con enfoque retrospectivo, de abordaje cuantitativo, cuyos datos fueron recolectados de las historias clínicas de 78 pacientes atendidos en julio y agosto de 2012, en el servicio de urgencias del Hospital Otávio de Freitas, en Recife, Pernambuco. Las quejas que motivan a los pacientes a buscar los servicios de urgencia pueden ser diversas e inespecíficas en relación con la patología renal, pero la investigación tras la admisión indica el grado de lesión y muestra la necesidad de diálisis. Un grupo significativo ya llega a los servicios de emergencia con pérdida de la función renal o en una fase terminal de la enfermedad y necesita un trasplante de riñón.

Palabras Clave: Enfermería; nefrología; urgencias; insuficiencia renal.

\section{INTRODUÇÃO}

As complicações das nefropatias são causas frequentes de atendimento nos serviços de emergência. São poucos os serviços de emergência especializada ou que possuem especialistas disponíveis para lidar com estas intercorrências. O tema é ainda pouco pesquisado pela enfermagem ${ }^{1,2}$.

As nefropatias são causas frequentes de admissão e internamento nos serviços de saúde. O diagnóstico, escolha do método dialítico, preparo do paciente e acesso venoso adequado precoces são decisivos como intervenções eficientes, evitando descompensações graves da homeostase. As unidades de saúde que prestam atendimento de urgência e emergência geral devem estar preparadas para atender a esse público específico, com protocolos bem estabelecidos e recursos humanos e materiais adequados. 
Sendo assim, apesar de os pacientes, muitas vezes, não compreenderem que suas queixas são de origem renal e recorrerem à emergência geral para atendimento e, considerando que elas são, com frequência, pouco específicas, há necessidade de se investigar mais tais eventos e divulgar evidências que sirvam de alerta aos profissionais, a fim de direcionar o planejamento dos serviços de saúde.

Objetivou-se, por meio deste estudo, descrever o perfil sociodemográfico e clínico dos indivíduos atendidos em uma emergência geral com insuficiência renal aguda (IRA).

\section{REVISÃo DE LITERATURA}

A incidência de insuficiência renal aguda tem crescido de forma acentuada nos últimos anos em consequência de dois mecanismos: aumento da prevalência da doença renal crônica e progresso nos procedimentos diagnósticos e terapêuticos ${ }^{3}$. A associação entre as duas entidades nosológicas, aguda e crônica, tem sido alvo de investigações que favoreçam a compreensão do fenômeno. As intervenções e diagnóstico precoces podem trazer melhores resultados, considerando-se que a descoberta da doença na fase aguda poderia ser evitada com o rastreamento bem feito ${ }^{3}$.

Os sinais e sintomas iniciais da IRA são inespecíficos, dificultando o diagnóstico precoce ${ }^{4}$. Em sua fase inicial, quando as manifestações clínicas e laboratoriais são mínimas ou ausentes, o diagnóstico pode ser sugerido pela associação de manifestações inespecíficas (fadiga, anorexia, emagrecimento, prurido, náusea ou hemólise, hipertensão, poliúria, nictúria, hematúria ou edema). Em consequência dos quadros de oligúria e anúria, as funções principais dos rins, ou seja, a manutenção do equilíbrio hidroeletrolítico e acidobásico e excreção dos produtos nitrogenados estão comprometidas, repercutindo em diversos órgãos e sistemas ${ }^{4,5}$.

O termo uremia descreve o processo de intoxicação e está associado a diversos e heterogêneos sintomas, incluindo prurido, manifestações neurológicas, náusea e vômito, diarreia, perda de apetite com anorexia, insônia e arritmias. Além disso, os pacientes urêmicos têm maior risco de infecções e complicações hematológicas, sendo mandatório o tratamento dialítico ${ }^{4}$.

Os motivos para a internação dos pacientes incluem causas renais e sistêmicas associadas, como hipertensão arterial, diabetes mellitus, lúpus e infecções ${ }^{6}$. O número de novos casos estimado para a Região Nordeste, em 2011, foi de 6001 pacientes e vem aumentando a taxa de incidência ao longo dos anos ${ }^{7}$. Cabe ressaltar que o enfermeiro é responsável pelo rastreamento e acompanhamento de pacientes portadores de diabetes e hipertensão arterial, principais causas de doença renal, na atenção básica ${ }^{8}$.

O impacto da instalação da doença em idosos é potencializado pelas comorbidades do envelhecimento ou é sua consequência9. Um estudo realizado em um serviço de nefrologia do Estado do Rio de Janeiro indica que $26,6 \%$ das internações ocorreram por complicações pós-transplantes ${ }^{10}$. Outro estudo, ao acompanhar a evolução da insuficiência renal em unidades de terapia intensiva, referiu que a intervenção precoce para pacientes críticos estava associada a melhor desfecho ${ }^{5}$.

Atualmente, em virtude das opções de tratamento dialítico contínuo e não contínuo, a enfermagem especializada deve estar habilitada a cuidar de pacientes submetidos a tratamento dialítico ${ }^{11}$. Contudo, no contexto do atendimento inicial, é importante que enfermeiros generalistas e emergencistas estejam aptos a reconhecer precocemente sinais sugestivos de IRA e tenham conhecimento sobre a abordagem terapêutica e a repercussão sistêmica e, sobremaneira, o manejo dos acessos vasculares. Um estudo de coorte recente revelou que pacientes acompanhados por enfermeiros especialistas em consultas de enfermagem periódicas apresentaram menos complicações, menos atendimentos de emergência e menos internamentos hospitalares ${ }^{12}$.

\section{Metodologia}

O estudo apresentado foi realizado no Serviço de Emergência Geral do Hospital Otávio de Freitas da Secretaria de Estado de Saúde de Pernambuco. Desde 2007, o serviço possui acolhimento e classificação de risco realizado por enfermeiros habilitados. Além disso, o serviço conta com duas enfermeiras destinadas à hemodiálise na emergência, com seis leitos cada. Para o internamento, os pacientes nefropatas são admitidos em outra enfermaria, com 16 leitos. $\bigcirc$ atendimento é de responsabilidade de uma clínica especializada terceirizada.

Trata-se de um estudo descritivo, retrospectivo, de abordagem quantitativa. Foram coletados dados de 78 prontuários de pacientes atendidos em julho e agosto de 2012. No período, foram atendidos 86 casos, dos quais oito não foram incluídos por falta de preenchimento de dados ou preenchimento incorreto, o que dificultaria a análise. Para orientar a coleta dos dados, foi elaborado um instrumento próprio, adequado à fonte de dados e ao objetivo da pesquisa, contendo dados socioeconômicos e clínicos epidemiológicos. A coleta de dados ocorreu após apreciação e aprovação do Comitê de Ética em Pesquisa do próprio hospital - CAAE 04015612.0.0000.5200 -, Parecer CEP HOF 54385.

A partir dos dados coletados, os níveis pressóricos foram estratificados conforme a VI Diretrizes Brasileiras de Hipertensão arterial ${ }^{13}$. A função renal foi avaliada segundo o critério da Acute Dialysis Quality Iniciative (ADQI) que desenvolveu, em 2002, o acrônimo RIFLE que define três graduações de gravidade de disfunção renal - Risco (classe R), Injúria (classe I) e Falência (classe F), baseadas na mudança da creatinina sérica e diurese - e duas classes de evolução (Perda classe L - e Estágio Final - classe E) ${ }^{14}$.

Os dados foram analisados com recursos de epidemiologia descritiva (frequência absoluta, frequência relativa etc.) usando-se o software estatístico Epi-info e apresentados em tabelas. 


\section{Resultados}

No total de 78 prontuários investigados, a média de idade alcançou $57,65 \pm 18,06$ anos, com predominância do sexo masculino - 54 (69,23\%) - e procedência da região metropolitana do Recife-PE. Apenas 10(12,8\%) pacientes já estavam em tratamento dialítico, sendo a média do tempo de tratamento 21,61 $\pm 16,44$ meses, de acordo com o exposto na Tabela 1.

TABELA 1: Características sociodemográficas e clínicas na admissão dos pacientes atendidos com insuficiência renal em emergência. Recife, 2012. ( $N=78)$

\begin{tabular}{|c|c|c|}
\hline Variáveis & $f$ & $\%$ \\
\hline \multicolumn{3}{|l|}{ Idade } \\
\hline$<20$ anos & 2 & 2,56 \\
\hline 20-35 anos & 2 & 2,56 \\
\hline $35-50$ anos & 23 & 29,49 \\
\hline $50-65$ & 25 & 32,05 \\
\hline $65-80$ & 19 & 24,37 \\
\hline$>80$ & 7 & 8,97 \\
\hline \multicolumn{3}{|l|}{ Sexo } \\
\hline Masculino & 54 & 69,23 \\
\hline Feminino & 24 & 30,77 \\
\hline \multicolumn{3}{|l|}{ Procedência } \\
\hline Capital & 7 & 8,97 \\
\hline RMR & 43 & 55,13 \\
\hline Interior & 28 & 35,90 \\
\hline \multicolumn{3}{|l|}{ Tratamento dialítico prévio } \\
\hline Sim & 10 & 12,82 \\
\hline Não & 68 & 87,18 \\
\hline \multicolumn{3}{|l|}{ Estado de hidratação } \\
\hline Hidratado & 49 & 62,82 \\
\hline Desidratado & 28 & 35,90 \\
\hline Edema & 1 & 1,28 \\
\hline \multicolumn{3}{|l|}{ Nível pressórico na admissão } \\
\hline Hipotensão & 11 & 14,10 \\
\hline Normal & 3 & 3,85 \\
\hline Limítrofe & 2 & 2,56 \\
\hline Hipertensão Grau I & 45 & 57,69 \\
\hline Hipertensão Grau II & 16 & 20,52 \\
\hline Hipertensão Grau III & 1 & 1,28 \\
\hline \multicolumn{3}{|l|}{ Padrão de eliminação urinária } \\
\hline Anúria & 24 & 30,78 \\
\hline Oligúria & 25 & 32,05 \\
\hline Disúria & 25 & 32,05 \\
\hline Poliúria & 2 & 2,56 \\
\hline Sem registro & 2 & 2,56 \\
\hline \multicolumn{3}{|l|}{ Via de eliminação urinária } \\
\hline Espontânea & 54 & 69,23 \\
\hline Sonda Vesical de Demora & 15 & 19,23 \\
\hline Dispositivo urinário & 3 & 3,85 \\
\hline Fralda & 4 & 5,13 \\
\hline Citostomia & 2 & 2,56 \\
\hline \multicolumn{3}{|l|}{ Aspecto da diurese } \\
\hline Normal & 21 & 26,93 \\
\hline Concentrado & 25 & 32,05 \\
\hline Hematúria & 31 & 39,74 \\
\hline Colúria & 1 & 1,28 \\
\hline
\end{tabular}

Na amostra, 49(62,80\%) apresentaram estado de hidratação sem alterações, sendo que apenas um paciente revelou edema. Quanto aos níveis pressóricos, 45(57,69\%) apresentavam hipertensão de grau I na admissão, 16(20,50\%) hipertensão de grau II e apenas um paciente com hipertensão de grau III. Foram classificados como hipotensos, na admissão, 11(14,10\%) indivíduos, conforme indicado na Tabela 1.

Quanto à eliminação urinária, 25(32,05\%) apresentavam oligúria, 24(30,78\%) anúria, e apenas $2(2,56 \%)$, poliúria, sendo a maioria com diurese espontânea - 54 (69,20\%) - e uso de fraldas ou dispositivo urinário. Foi registrado urina com aspecto concentrado em 25(32,10\%) dos casos e hematúria em 31(39,74\%), conforme Tabela 1.

Quanto ao estadiamento da insuficiência renal, considerando o volume de diurese e a dosagem sérica de creatina dos pacientes, $19(24,37 \%)$ foram classificados com lesão renal e 30(38,46\%), com falência renal. Dos pacientes, apenas $2(2,56 \%)$ apresentavam perda da função renal e $12(15,38 \%)$ com doença renal em estágio terminal, de acordo a Tabela 2.

Após a admissão e o diagnóstico médico com indicação de terapia renal substitutiva, para início do tratamento, a maioria recebeu cateter central para hemodiálise com triplo lúmen, implantado principalmente em veia jugular interna direita em $23(29,5 \%)$ e esquerda em 14(17,9\%), fossa ilíaca direita de $20(25,6 \%)$ e esquerda de $10(12,8 \%)$ pacientes. A fístula arteriovenosa previamente confeccionada foi utilizada para o tratamento de 2(2,60\%) pacientes que já estavam em tratamento dialítico, conforme a Tabela 2.

TABELA 2: Distribuição dos pacientes quanto à classificação da insuficiência renal e acessos vasculares utilizados para a terapia dialítica. Recife-PE, 2012. ( $N=78)$

\begin{tabular}{lcc}
\hline Variáveis & $\mathrm{f}$ & $\%$ \\
\hline Classificação & $\mathrm{n}$ & $\%$ \\
Risco de disfunção renal & 15 & 19,23 \\
Lesão renal & 19 & 24,37 \\
Falência da função renal & 30 & 38,46 \\
Perda da função renal & 2 & 2,56 \\
Doença renal em estágio terminal & 12 & 15,38 \\
Tipo de cateter & & \\
Prévio & 10 & 12,82 \\
Central & 2 & 2,56 \\
Fístula arteriovenosa & 2 & 2,56 \\
Central p/ HD 2 lúmen & 1 & 1,30 \\
Central p/ HD 3 lúmen & 63 & 80,77 \\
Local de implantação do cateter & & \\
Fossa ilíaca direita & 20 & 25,64 \\
Fossa ilíaca esquerda & 10 & 12,82 \\
Membro superior direito & 3 & 3,85 \\
Membro superior esquerda & 1 & 1,30 \\
Veia jugular interna direita & 23 & 29,50 \\
Veia jugular interna esquerda & 14 & 17,90 \\
Veia subclávia direita & 4 & 5,13 \\
Veia subclávia esquerda & 2 & 2,56 \\
Sem registro & 1 & 1,30 \\
\hline
\end{tabular}


As queixas principais que motivaram os pacientes a buscar a emergência foram: dor abdominal $24(30,76 \%)$, dispneia $12(15,38 \%)$, febre $12(15,38 \%)$ e disúria $10(12,82 \%)$, como mostra a Tabela 3.

TABELA 3: Queixas principais que motivaram os pacientes em estudo a buscarem o serviço de emergência. Recife-PE, 2012

\begin{tabular}{lcc}
\hline Variáveis & $\mathrm{f}$ & $\%$ \\
\hline Queixa principal(*) & & \\
Dor abdominal/flanco & 24 & 30,76 \\
Dispneia & 12 & 15,38 \\
Febre & 12 & 15,38 \\
Disúria & 10 & 12,82 \\
Vômito & 9 & 11,54 \\
Náusea & 5 & 6,41 \\
Desorientação & 4 & 5,13 \\
Diarreia & 2 & 2,56 \\
Crise hipertensiva & 3 & 3,85 \\
Edema & 2 & 2,56 \\
Queixas inespecíficas/não sugestivas & 43 & 55,13 \\
Hipótese diagnóstica(*) & & \\
IRC (fase aguda) & 36 & 46,15 \\
IRA & 19 & 24,37 \\
Hipertensão arterial sistêmica & 12 & 15,38 \\
Infecção do trato urinário & 8 & 10,26 \\
Trauma & 8 & 10,26 \\
Nefrolitíase & 4 & 5,13 \\
Diabetes & 3 & 3,85 \\
Obstrução intestinal & 2 & 2,56 \\
Outros & 26 & 33,33 \\
\hline
\end{tabular}

(*)Respostas múltiplas

As hipóteses diagnósticas médicas na admissão dos pacientes foram: IRA 19(24,37\%), insuficiência renal crônica 36(46,15\%), hipertensão arterial 12(15,38\%), infecção do trato urinário 8(10,26\%). Houve $1(12,38 \%)$ paciente que apresentou IRA quando realizada terapia antirretroviral.

\section{Discussão}

A detecção precoce do prejuízo da função renal de forma aguda nos atendimentos de emergência pode desencadear medidas mais efetivas na proteção do paciente, diminuindo tempo de permanência no hospital e complicações ${ }^{15}$.

A maioria dos pacientes apresentava, no momento da admissão, o primeiro quadro indicativo de lesão renal ou desconheciam a insuficiência crônica. Os pacientes que já haviam recebido o diagnóstico tiveram média menor que dois anos para apresentar as queixas que caracterizam situação de urgência, mesmo já estando em tratamento dialítico.

Um estudo realizado em um serviço de emergência da Inglaterra relata que 25,4\% dos pacientes admitidos apresentaram insuficiência renal aguda durante a permanência no hospital, sendo que um terço deles já apresentavam sinais, sintomas e achados laboratoriais que permitiram o diagnóstico na chegada ao serviço ${ }^{15}$. Os pacientes pesquisados neste estudo mostravam maior índice de falência renal $(38,46 \%)$ que os investigados na Inglaterra $(11,67 \%)$, o que provavelmente está associado ao cuidado prévio na atenção primária à saúde ${ }^{15}$.

A média de idade dos pacientes nesta investigação e a prevalência maior do sexo masculino corroboram com outros estudos ${ }^{16-21}$. A distribuição das idades não apresentou diferença significativa entre idosos e adultos.

Os níveis pressóricos e o estado de hidratação corroboram com os achados em outras publicações 9,17-21 $^{\text {. }}$ Um estudo realizado com pacientes de terapia intensiva revelou $68,6 \%$ de causas pré-renais para pacientes sem indicação de terapia dialítica, sendo a principal a hipertensão arterial $^{22}$. A hipertensão arterial é um dos principais fatores de risco modificáveis para várias doenças, impulsionando o grupo das doenças cardiovasculares como principal causa de morte no país ${ }^{13}$. Em 2011, a hipertensão foi tida como principal doença de base para os pacientes em tratamento dialítico no país $(35,1 \%)$, seguida pela diabetes $(28,4 \%)^{7}$.

A oligúria e a anúria, apesar de não terem sido as principais queixas referidas pelos pacientes, teve elevada incidência na amostra, como apresentado por outras pesquisas ${ }^{17}$. $\mathrm{O}$ volume de diurese é o primeiro critério para avaliação da função renal, sendo importante para o estadiamento, independente da taxonomia adotada e devendo ser controlada com rigor ${ }^{14}$. A redução do volume de diurese está imbricada à retenção de fluidos, a qual pode agravar a hipertensão e insuficiência cardíaca, o que pode progredir para o edema pulmonar ${ }^{4}$.

Apesar de não ter sido avaliada estatisticamente, as queixas principais mais apresentadas podem ser relacionadas, clinicamente, à alteração do equilíbrio hidroeletrolítico, mais especificamente aos níveis de sódio e potássio e alteração do pH do sangue em função do acúmulo de excretas nitrogenadas. As queixas principais mostravam, também, relação com outros quadros clínicos inespecíficos, o que sugere mais estudos epidemiológicos da associação entre o quadro renal agudo e outras doenças de base que o possam ocasionar (Tabela 3). Em particular, a insuficiência cardíaca, doenças da aorta e o quadro de sepse, que atinge amplamente os idosos, podem repercutir em lesão renal ${ }^{4}$.

Um estudo internacional avaliou, de forma retrospectiva, 5680 atendimentos de emergência, dos quais 992 apresentaram sepse e choque séptico. Desses pacientes, 57,7\% desenvolveu IRA, estando associado à idade avançada, doença renal preexistente, leucopenia e plaquetopenia. $\bigcirc$ grupo que desenvolveu o quadro renal agudo manifestou, também, maior mortalidade e tempo de internamento ${ }^{23}$. 
Quanto aos acessos vasculares, outro estudo, avaliando 23 clínicas distribuídas em sete estados brasileiros, revelou que, para os pacientes com insuficiência renal crônica, as fístulas arteriovenosas eram a escolha para $93,4 \%{ }^{24}$. No entanto, devido ao caráter emergencial da necessidade de tratamento versus prazo necessário à maturação da fístula, os cateteres foram preteridos em $97,4 \%$ dos casos. Tal estudo apontou que, no nordeste do país, $47 \%$ dos cateteres foram implantados em veia jugular interna e 33\% em veia subclávia ${ }^{24}$.

A amostra teve acessos implantados, em ordem de incidência, nas veias jugulares, femorais e subclávias; contudo, outros autores apontam que as veias subclávias são preferidas em virtude da maior facilidade na colocação do cateter ${ }^{24-26}$. Estudos mostram que o risco de infecção aumenta significativamente em cateter venoso central, inserido em veia jugular interna em relação à inserção em veia subclávia que, por sua vez, está associada a risco maior de complicações não infecciosas (pneumotórax, hemotórax, trombose venosa) ${ }^{4,26}$. Apesar de não ser utilizada ainda nos serviços brasileiros, estudos revelam que pode haver benefícios na utilização precoce de diálise peritoneal na IRA ${ }^{27}$.

Embora não tenha sido avaliado neste estudo, outra investigação nacional, conduzida com 129 pacientes de trauma admitidos em um serviço de emergência, revelou que 52(40,31\%) deles evoluíram para IRA, dos quais 19(36,5\%) precisaram de diálise. Nessa amostra, todos os pacientes com IRA secundário ao trauma evoluíram para óbito ${ }^{28}$. Na Inglaterra, um inquérito com 765 pacientes de serviços de emergência geral apontou que a IRA é mais frequente do que a literatura existente sugere, chegando a $25 \%$ dos casos de admissões e está associada a aumento no tempo de internamento, chance de admissão em leitos de terapia intensiva e risco de morte ${ }^{15}$. Outra publicação, comparando pacientes que alcançaram a resolução do quadro de IRA em até 5 dias com pacientes em que a duração da IRA adquirida no hospital foi maior que 6 dias, revelou que o aumento no tempo de resolução do quadro aumentou significativamente o risco de morte durante o internamento e no período pós-alta acompanhando por até 240 dias $^{29}$.

\section{Conclusão}

É útil a compreensão das manifestações da lesão renal pelo enfermeiro emergencista, em especial aos que realizam triagem, acolhimento e classificação de risco. As queixas que motivam os pacientes a procurarem a emergência podem ser diversas e inespecíficas em relação à patologia renal, mas a investigação após a admissão acaba indicando o grau de lesão e a necessidade de tratamento dialítico. Muitos pacientes procuram a emergência e recebem diagnóstico de insuficiência renal, visto que não apresentavam previamente doença renal crônica conhecida. Um grupo significativo chega aos serviços de emergência já com perda da função renal ou em estágio terminal de doença e necessidade de transplante renal.

No tocante à assistência prestada, os serviços de saúde com atendimento de emergência devem reconhecer a necessidade de oferecer o tratamento dialítico com presteza, buscando melhorar o prognóstico. Os estabelecimentos de saúde devem se organizar em rede para ofertar, no sistema de referência e contrarreferência, a atenção necessária e cada unidade deve garantir o suprimento de cateteres e materiais de qualidade.

O estudo teve por limitação o intervalo disponível para coleta, que restringiu a amostra avaliada. Além disso, retrata a realidade de apenas um serviço. Em estudos posteriores, devem ser avaliados os fatores de risco e outras causas que possam estar relacionadas ao evento.

\section{REFERÊNCIAS}

1.Carvalho GMC, Lima FET, Barbosa IV, Melo EM. Estudos brasileiros sobre nefrologia nas teses e dissertações de enfermagem. Rev Bras Enferm. 2010 [citado em 31 jul 2015] 63:1052-3. Disponível em: http://www. scielo.br/scielo.php?script $=$ sci_arttext $\&$ pid $=$ S0034 $71672010000600028 \& \operatorname{lng}=$ pt. http://dx.doi. org/10.1590/S0034-71672010000600028.

2.Pennafort VPS, Furtado AM, Fialho AVM, Moreira TMM, Freitas MC, Queiroz MVO. Produção do conhecimento científico de Enfermagem em Nefrologia. Rev Bras Enferm. 2010 [citado em 31 jul 2015] 63:830-6. Disponível em: http://www.scielo.br/scielo.php?script =sci_ pdf\&pid $=$ S0034-71672010000500022 \&lng =en\&nrm $=$ iso $\&$ tlng $=$ pt.

3.Schmidt JJ, Beutel G, Kielstein JT. Diagnosis and pathophysiology of acute renal failure - is prevention possible? Dtsch Med Wochenschr. 2015 [citado em 31 jul 2015]; 140:245-9. Disponível em: http://www.ncbi. nlm.nih.gov/pubmed/25704519.

4.Patschan D, Muller GA. Acute kidney injury. J Inj Violence Res. 2015 [citado em 31 jul 2015]; 7(1):19. 26. Disponível em: http://www.ncbi.nlm.nih.gov/pmc/ articles/PMC4288292/pdf/jivr-07-19.pdf.

5.Silva VTC. Insuficiência renal aguda em unidade de tratamento intensivo: perfil epidemiológico e validação de índices prognósticos [tese de doutorado] São Paulo: Universidade de São Paulo; 2007.

6.Oliveira FC, Alves MDS, Bezerra AP. Co-morbidades e mortalidade de pacientes com doença renal: atendimento terceirizado de nefrologia. Acta Paul Enferm. 2009 [citado em 31 jul 2015] 22:476-80 Disponível em: http://www.scielo.br/pdf/ape/v22nspe1/03.pdf.

7.Sesso RCC, Lopes AA, Thomé FS, Lugon JR, Watanabe Y, Santos DR. Diálise Crônica no Brasil Relatório do Censo Brasileiro de Diálise, 2011. J Bras Nefrol. 2012 [citado em 31 jul 2015] 34:272-7. Disponível em: http://www.scielo.br/scielo.php?script =sci_ arttext $\&$ pid $=$ S0101-28002012000300009\&lng $=$ pt. http://dx.doi.org/10.5935/0101-2800.20120009.

8. Travagim DSA, Kusumota L, Teixeira CRS, Ce- 
sarino CB. Prevenção e progressão da doença renal crônica: atuação do enfermeiro com diabéticos e hipertensos. Rev enferm UERJ 2010 [citado em 31 jul 2015] 18:291-7. Disponível em: http://www.revenf. bvs.br/scielo.php?script $=$ sci_arttext $\&$ pid $=$ S0104 $35522010000200021 \& \operatorname{lng}=$ pt.

9.Ribeiro DF. O cuidador do idoso com insuficiência renal crônica em diálise peritoneal ambulatorial contínua [dissertação de mestrado] Ribeirão Preto (SP): Universidade de São Paulo; 2008.

10.Ribeiro RCHM; Garcia TPR; Bertolin DC; Cesarino CB; Poletti NAA; Rodrigues AMS. Pacientes vítimas de politrauma com insuficiência renal aguda na Unidade de Terapia Intensiva. Acta Paul Enferm. 2008 [citado em 31 jul 2015] 21:216-20. Disponível em http://www. scielo.br/scielo.php?script $=$ sci_pdf\&pid $=$ S0103 $21002008000500015 \& \operatorname{lng}=\mathrm{en} \& n r m=$ iso $\&$ tlng $=$ pt.

11.Araujo STC; Koeppe GBO. Setor de nefrologia: uma parceria entre ensino e service. Acta Paul Enferm. 2012 [citado em 31 jul 2015] 22:572-6. Disponível em: http:// www.scielo.br/pdf/ape/v22nspe1/26.pdf.

12.Lee DTF, Choi CK, Chan CW, Chair SY, Chan D, Fung SYK et al. The impact on patient health and service outcomes of introducing nurse consultants: a historically matched controlled study. BMC Health Services Research. 2013 [cited in 2015 Mar 9]; 13:431. Available from: http://www.biomedcentral.com/1472-6963/13/431 13.Sociedade Brasileira De Cardiologia, Sociedade Brasileira De Hipertensão, Sociedade Brasileira De Nefrologia. VI Diretrizes Brasileiras de Hipertensão. Arq bras cardiol. 2010 [citado 22 jan 2013] 95(1):I-III. Disponível em: http://www.scielo.br/scielo.php?script = sci arttext\&pid $=$ S0066-782X2010001700001\&lng $=$ pt. http://dx.doi.org/10.1590/S0066-782X2010001700001. 14.Kim MH, Koh SO, Kim EJ, Cho JS, Na SW. Incidence and outcome of contrast-associated acute kidney injury assessed with Risk, Injury, Failure, Loss, and End-stage kidney disease (RIFLE) criteria in critically ill patients of medical and surgical intensive care units: a retrospective study. BMC Anesthesiol. 2015 [cited in 9 Mar 2015]; 15:23. Available from: http://www.ncbi.nlm.nih.gov/ pmc/articles/PMC4360925/.

15.Challiner R, Ritchie JP, Fullwood C, Loughnan P, Hutchison A. Incidence and consequence of acute kidney injury in unselected emergency admissions to a large acute UK hospital trust. BMC Nephrology. 2014 [citado em 9 mar 2015]; 15:84. Disponível em: http:// www.biomedcentral.com/1471-2369/15/84.

16.Santos ER. Lesão renal aguda em terapia intensiva avaliada pelo RIFLE. Rev bras ter intensiva. 2009 [citado em 31 jul 2015]; 21:359-68. Disponível em: http://www. scielo.br/pdf/rbti/v23n3/v23n3a10.pdf

17.Silva Júnior GB, Daher EF, Mota RMS, Menezes FA. Risk factors for death among critically ill patients with acute renal failure. Sao Paulo Med J. 2006 [cited in $2015 \mathrm{Jul} 31$ ]; 124(5):257-63. Available from: http:// www.scielo.br/pdf/spmj/v124n5/04.pdf.

18.Balbi AL, Gabriel DP, Barsante RC, Caramori JT, Martin LC, Barreti P. Mortalidade e prognóstico específico em pacientes com insuficiência renal aguda. Rev Assoc Med Bras. 2005 [citado em 31 jul 2015]; 51:318-22. Disponível em: http://www.scielo.br/pdf/ramb/v51n6/27532.pdf.
19.Pinto OS, Carminatti M, Lacet T, Rodrigues DF, Nogueira LO, Bastos MG et al. Insuficiência renal aguda nefrotóxica: prevalência, evolução clínica e desfecho. J Bras Nefrol 2009 [citado em 31 jul 2015]; 31:183-9. Disponível em: http:/www.scielo.br/pdf/jbn/v31n3/v31n3a03.pdf. 20.Silvester W, Bellomo R, Cole L. Epidemiology, management, and outcome of severe acute renal failure of critical illness in Australia. Crit Care Med. 2001 [cited in 2015 Jul 31]; 29:19105. Disponível em: http://www. ncbi.nlm.nih.gov/pubmed/11588450.

21.Xavier BLS, Santos I, Almeida RF, Clos AC, Santos MT. Características individuais e clínicas de clientes com doença renal crônica em terapia renal substitutiva. Rev enferm UERJ. 2014 [citado em 31 jul 2015]; 22:314-20 Disponível em: http://www.e-publicacoes.uerj.br/index. php/enfermagemuerj/article/view/13683/10474.

22.Bernardina LC, Diccini S, Belasco AGS, Bittencourt ARC, Barbosa DA. Evolução clínica de pacientes com insuficiência renal aguda em unidade de terapia intensiva. Acta Paul Enferm. 2008 [citado em 31 jul 2015] 21:174-8. Disponível em http:/www.scielo.br/pdf/ape/v21nspe/a07v21ns.pdf. 23.Sang Heon Suh, Chang Seong Kim, Joon Seok Choi, Eun Hui Bae, Seong Kwon Ma, Soo Wan Kim. Acute kidney injury in patients with sepsis and septic shock: risk factors and clinical outcomes. Yonsei Med J. 2013 [cited in 2015 Jul 31]; 54:965-72. Available from: http://www.ncbi.nlm. nih.gov/pmc/articles/PMC3663224/pdf/ymj-54-965.pdf.

24.Linardi F, Linardi FF, Bevilacqua JL, Morad JFM, Costa JA, Miranda Júnior F. Acesso vascular para hemodiálise: avaliação do tipo e local anatômico em 23 unidades de diálise distribuídas em sete estados brasileiros. Rev col bras cir. 2003 [citado em 31 jul 2015] 30(3) 183-93. Disponível em http://www.scielo.br/pdf/rcbc/v30n3/a05v30n3.pdf.

25.Nasir Mahmood S, Naveed Mukhtar K, Iqbal N, Umair SF. Pre dialysis care and types of vascular access employed in incident hemodialysis patients: A study from Pakistan. Pak J Med Sci. 2013 [cited in 2015 Jul 31] 29:828-31. Disponível em: http://www.ncbi.nlm.nih. gov/pmc/articles/PMC3809299/pdf/pjms-29-828.pdf.

26. Grothe C, Belasco AGS, Bittencourt ARC, Vianna LAC, Sesso RCC, Barbosa DA. Incidência de infecção da corrente sanguínea nos pacientes submetidos à hemodiálise por cateter venoso central. Rev Latino-Am Enfermagem. 2010 [citado em 31 jul 2015] 18(1) Disponivel em http://www.scielo.br/pdf/rlae/v18n1/pt_12.pdf. 27.Yong K, Dogra G, Buodville N, Pinder M, Lim W. Acute kidney injury: controversies revisited. Int J Nephrol. 2011 [cited in 2015 Jul 31] 2011:762634. Disponível em: http:/www.ncbi.nlm.nih.gov/pmc/articles/ PMC3108161/pdf/IJN2011-762634.pdf.

28.Abreu KLS, Silva Júnior GB, Barreto AGC, Melo FM, Oliveira BB, Mota RMS et al. Acute kidney injury after trauma: prevalence, clinical characteristics and RIFLE classification. Indian J Crit Care Med. 2010 [cited in 2015 Jul 31]; 14(3): 121-8. Available from: http://www. ncbi.nlm.nih.gov/pmc/articles/PMC3021827/.

29. Yoo J, Lee JS, Lee J, Jeon JS, Noh H, Han DC, Kwon $\mathrm{SH}$. Relationship between duration of hospital-acquired acute kidney injury and mortality: a prospective observational study. Korean J Intern Med. 2015 [cited in 2015 Jul 31]; 30:205-11. Available from: http://www.ncbi.nlm. nih.gov/pubmed/25750562. 